\title{
Patterns of retrograde axonal degeneration in the visual system
}

\author{
Willemien A. de Vries-Knoppert ${ }^{1}$ Johannes C. Baaijen, MD ${ }^{2}$ \\ Axel Petzold MD PhD ${ }^{3}$ \\ doi: 10.1093/brain/awz221
}

\footnotetext{
${ }^{1}$ Dutch Expertise Centre for Neuro-ophthalmology \& Department of Ophthalmology, Amsterdam UMC, Vrije Universiteit Amsterdam, The Netherlands

${ }^{2}$ Amsterdam UMC, Vrije Universiteit Amsterdam, Department of Neurosurgery, De Boelelaan 1117, Amsterdam, The Netherlands

${ }^{3}$ Dutch Expertise Centre for Neuro-ophthalmology \& Department of Neurology, Amsterdam UMC, Amsterdam, The Netherlands; Moorfields Eye Hospital \& The National Hospital for Neurology and Neurosurgery, London, UK; UCL Queen Square Institute of Neurology, London, UK; ORCiD:0000-0002-0344-9749.|URL@UCL.Email: a.petzold@ucl.ac.uk
} 


\begin{abstract}
Conclusive evidence for existence of acquired retrograde axonal degeneration which is truly trans-synaptic (RTD) has not yet been provided for the human visual system. Convincing data relies on experimental data of lesions to the posterior visual pathways. This study aimed to overcome the limitations of previous human studies, namely pathology to the anterior visual pathways and neurodegenerative co-morbidity. In this prospective, longitudinal cohort retinal OCT scans were acquired before and after elective partial temporal lobe resection in 25 patients for intractable epilepsy. Newly developed region of interest (ROI) specific, retinotopic areas substantially improved on conventional reported ETDRS grid based OCT data. Significant inner retinal layer atrophy separated patients with normal visual fields from those who developed a visual field defect. Acquired RTD affected the retinal nerve fibre layer (RNFL), ganglion cell (GCL) and inner plexiform layer (IPL) and stopped at level of the inner nuclear layer (INL). There were significant correlations between the resected brain tissue volume and the GCL-ROI $(R=-0.78, p<0.0001)$ and GCIPL-ROI $(R=-0.65$, $p=0.0007)$. In one patient damage to the anterior visual pathway resulted in occurrence of microcystic macular oedema (MMO) as recognised from experimental data. In the remaining 24 patients with true RTD, atrophy rates in the first three months were strongly correlated with time form surgery for the GCL-ROI $(R=-0.74, p<0.0001)$ and the GCIPL-ROI $(R=-$ $0.51, p<0.0001)$. The different time course of atrophy rates observed relate to brain tissue volume resection and suggest that three distinct patterns of retrograde axonal degeneration exist: (1) direct retrograde axonal degeneration, (2) rapid and self terminating RTD, (3) prolonged RTD representing a "penumbra" which slowly succumbs to molecularly governed spatial cellular stoichiometric relationships. We speculate that the latter could be a promising target for neuroprotection.
\end{abstract}

Short running title: Retrograde trans-synaptic axonal degeneration

Keywords Meyers loop, neurodegeneration, optical coherence tomography, retinal layer segmentation, epilepsy surgery. 


\section{Contents}

$\begin{array}{lll}1 & \text { Introduction } & 4\end{array}$

2 Methods 5

\begin{tabular}{lll}
\hline 3 & Results & 8
\end{tabular}

4 Discussion 12

\begin{tabular}{ll}
\hline References & 19
\end{tabular}

\section{List of Figures}

$1 \quad$ Visual fields, OCT and MRI . . . . . . . . . . . . . 23

2 Region of interest (ROI) approach for OCT . . . . . . . . . 25

3 Longitudinal OCT data ETDRS based. . . . . . . . . . . . . 27

$4 \quad$ Longitudinal OCT data ROI based. . . . . . . . . . . . . . . . . . 29

5 Time course direct retrograde axonal degeneration . . . . . . . . 31

6 Time course in trans-synaptic retrograde axonal degeneration . . 33 


\section{Introduction}

In vivo evidence for presence of acquired retrograde trans-synaptic axonal degeneration (RTD) in the visual system is recent (Jindahra et al. 2009; Mehta and Plant 2005). This work has been reproduced (Goto et al. 2016) Park et al. 2013. Schwartz et al. 2017). But the true existence of RTD has still not been confirmed conclusively demonstrated because of an overlooked detail of the vascular supply of the visual system.

An important limitation of all above studies in patients with an ischaemic stroke of the middle (MCA) and posterior cerebral artery (PCA) territories (Goto et al. 2016 Jindahra et al. 2009 Park et al. 2013: Schwartz et al. 2017 ) is that a direct ischaemic insult to the optic tract and lateral geniculate nucleus (LGN) remains possible (Luco et al. 1992 Pula and Yuen 2017). Anatomically, the LGN and optic tract are supplied by the anterior and lateral choroidal arteries which branch from the distal internal carotid artery, the proximal MCA, and the PCA. In addition, a large proportion of patients suffering from an ischaemic stroke have a considerable vascular burden with the chance for concomitant ischaemic damage to the anterior visual pathways (Pula and Yuen 2017). Likewise, evidence for RTD from other diseases can be disputed on similar grounds on the full extend of the brain pathology. Finally, patients may not be aware of their visual deficit which makes it impossible to precisely time when the stroke had happened (Mehta and Plant 2005).

Taken together there remains the possibility that some of the Retinal Nerve Fibre Layer atrophy (RNFL) observed in previous studies (Goto et al. 2016. Jindahra et al. 2009: Park et al. 2013. Schwartz et al.2017) could be caused by direct retrograde axonal degeneration, rather than by RTD. To date the most robust evidence for RTD comes from experimental studies lesioning the posterior visual pathway (Buren 1963).

To address the important question on whether or not true acquired RTD ex- 
ists we have conducted the present study. We prospectively recruited patients undergoing surgery which can cause an isolated lesion to the posterior visual pathways. The advantages of this study design are several. First, a baseline assessment of visual function, retinal structure and integrity of the visual pathways is possible. Second, timing of the intervention is exactly known. Third, it overcomes key limitations of previous studies on vascular supply, co-morbidity and extensive neurodegenerative pathology.

\section{Methods}

Study design and patients This prospective single centre study was approved by the ethics committee of the Amsterdam University Medical Centre (location VUmc, protocol number 2012/443) and the scientific research committee (protocol number CWO/12-16F). Written, informed consent was obtained from all patients. Consecutive patients undergoing partial temporal lobe resection as planned surgery for medically intractable epilepsy were screened for inclusion. Investigations were part of an established multidisciplinary patient pathway for epilepsy surgery which includes assessment of the

visual system because of the well known risk to damage Meyer's loop during epilepsy surgery (Jeelani et al. 2010).

The only exclusion criterion was previous exposure to vigabatrin which can cause retinal layer atrophy (Clayton et al.2010. Clayton et al. 2011).

Visual function testing The best corrected high contrast Snellen visual acuity (BCVA) was recorded at baseline, three and twelve months post-surgery. Data are reported as digital values (Petzold et al. 2014). At each visit a Goldmann visual field was recorded for each eye by a trained orthoptist, carefully monitoring monocular fixation. All Goldmann visual fields were scanned for digital analysis. For each Goldmann visual field the cSLO image of the retina 
was overlaid such that the macula matched the central visual field and the optic disc fell on the blind spot (Fig. 1). Based on this match the area of VFD was outlined and a the corresponding retinal layer thicknesses were calculated from the OCT data.

Images were analysed on a LINUX workstation using the GNU Image Manipulation Program (GIMP is distributed under the GNU General Public License and can be obtained free of charge from www.gimp.org). The baseline isopters II and I 2 were taken as reference. The difference in area between baseline and three months follow up was marked. Based on this semi-quantitative data patients were classified in three groups; (1) patients without a visual field defect, (2) patients with a small visual field defect (less than a quadrant, $1 \mathrm{E}$ ) and (3) patients with a large visual field defect (more than a quadrant, 1 F).

Imaging Brain magnetic resonance imaging (MRI) was performed on a 3T whole body system (GE Signa HDxt, Milwaukee, WI, USA). Scans were obtained pre- and post-surgery using T1 and T2 weighted sequences. Each MRI was reviewed for damage to the optic nerves, chiasm, optic tracts and optic radiations on coronal and axial scans. Axial scans were used to measure the distance from the posterior border of the eye to the chiasm and optic tracts up to the site of partial temporal lobectomy. An exclusion criterion was evidence for damage to the LGN or optic tracts on imaging (Fig. 1 .

All OCT images were obtained with a SD-OCT (Heidelberg Spectralis, software version 1.1.6.3) with the eye tracking function enabled for best accuracy (Balk and Petzold 2013). Data were collected from a peripapillary ring scan (12 degrees, 1 B-scan) and a macular volume scan (20x20 degrees, 49 Bscans, ART=20). The right eye is indicated as "OD" and the left eye as "OS".

Image post processing Automated segmentation was performed with the manufacturers software (HEYEX version 1.10.2.0, Viewing Module version 
6.9.5.0). All scans underwent a rigorous quality control $(Q C)$ check (Schippling et al. 2015. Tewarie et al. 2012). All scans were reviewed for presence of microcystic macular oedema (MMO) (Gelfand et al. 2012). Algorithm failures were corrected by hand. The peripapillary retinal nerve fibre layer (pRNFL), macular ganglion cell inner plexiform layer (GCIPL) and macular inner nuclear layer (INL) were exported for statistical analysis.

Previous studies used the ETDRS grid for calculating of individual retinal layer thickness maps (Fig. 2). One important limitation of this approach is caused by the design of the ETDRS grid sectors. The ETDRS sectors are arranged such that they do not respect the vertical midline of the macula as relevant for analysis of the anatomical relationships key to this study. For example in a patient with a quadrantanopia the ETDRS grid averages data from the healthy and atrophied macular area (Fig. 2 left bottom). This results in masking of relevant localised atrophy data.

For this reason we performed two additional analyses using a region of interest (ROI) as summarised in Fig. 2. First, we defined a region of interest (ROI) from the visual field overlay as shown in Fig. 1 and detailed in the next paragraph. The pointer (Fig. 2 left top, green arrow) was used to average the $\mathrm{GCL}$ thickness data from this ROI. We only calculated the average GCL-ROI thickness for this ROI because this was done by hand and pixel wise match for the GCL and IPL was not reliable. We therefore only report the GCL data for this ROI. Second, we used a purpose build grid consisting of $8 \times 8$ squares was fitted over the macular volume scan (Fig. 2 left top). Each box gave the thickness of the GCL in $\mu \mathrm{m}$ and the volume. The area of the GCIPL from three diagonally adjacent squares into the area anatomically corresponding to the site of surgery was calculated (GCIPL-ROI).

Taken together, we report thickness data for the ETDRS-GCIPL, GCIPLROI and GCL-ROI. 
Statistical analysis The statistical analyses were performed in SAS (version 9.4). First, normality was tested graphically and using Shapiro-Wilk statistics. Non-parametric tests were used for non-normal or skewed data and parametric tests for normally distributed data. Respectively the median (interquartile range, IQR) or mean \pm standard deviation (SD) were shown.

Differences between two groups were analysed using the Chi-square test for categorical variables, the two-tailed t-test for parametric continuous variables and the Mann-Whitney $U$ test for non-parametric continuous variables. Correlation analyses were performed using Pearson's R for normally distributed and Spearman's R for non-Gaussian data. The Bonferroni method was used to correct for multiple correlations.

The differences for segmented retinal layer thickness data between groups was analysed using generalised estimation equations (GEE) as recommended (Cruz-Herranz et al.2016). The GEE were adjusted for intra subject inter-eye correlations, repeated measurements and employed an exchangeable correlation structure. A p-value of 0.05 was accepted as significant.

\section{Results}

The study cohort consisted of 10/25 (40\%) female patients. The median age was 40 years (range 20 to 64 years). The diagnosis for epilepsy was hippocampal sclerosis in 12 (48\%), ganglioglioma in 3 (13\%), cavernoma in 2 (8\%), a dysembryoplastic neuro ectodermal tumor in $2(8 \%)$, gliosis in $2(8 \%)$, focal dysplasia in 1 (4\%), mild cortical dysplasia in $1(4 \%)$ and in one case histology only showed unspecific reactive changes.

Prior to surgery patients were treated with the following anti-epileptic drugs (AED). Carbamazepine $(n=19)$, Levetiracetam $(n=11)$, Clobazam $(n=8)$, Lamotrigine $(n=6)$, Retigabine $(n=1)$, Zonisamide $(n=1)$, Oxcarbamazepine $(n=1)$, Sodium Valproate $(n=1)$, Lacosamide $(n=5)$, Clonazepam $(n=8)$, Phenytoin 
$(n=1)$, Pregabalin $(n=1)$, Topiramate $(n=2)$.

The time interval from the baseline neuro-ophthalmological assessment to surgery averaged at $0.9 \pm 1.1$ months. The time interval between surgery to the next neuro-ophthalmological assessment averaged at $4.7 \pm 3.3$ months. All 25 patients completed baseline and first follow up.

The time from surgery to the second follow up averaged at $11.6 \pm 2.2$ months. This follow up was attended by $14 / 25$ (56\%) patients. The remaining 11 patients were lost to neuro-ophthalmological follow-up either because they had no visual problem, preferred to be seen by their local ophthalmologist for logistic reasons or gave no reason.

The volume of brain tissue resectioned was $5.26 \pm 1.45 \mathrm{~cm}^{3}$. In 24/25 (96\%) of patients the optic tract and LGN were intact on imaging after surgery. The outcome of surgery was rated on the Engel outcome scale as $1 A(n=20)$, $1 B(n=2), 2 A(n=1), 2 B(n=2)$. And on the ILAE outcome scale as class 1 $(n=19)$, class $2(n=3)$ and class $3(n=3)$.

Visual function structure composite Fig. 1 summarises matching of visual function and structure in a patient with only a minimal VFD and a patient with a large quadrantanopia reaching the central visual field. In this cohort the visual field remained normal after surgery in 4/25 (16\%). A quadrantanopia was observed in 20/25 (70\%). The quadrantanopia was to the left in 11 (44\%) and right in 9 (36\%).

A right temporal hemianopia was observed in one patient. This patient suffered from damage to the optic tract during surgery. Therefore it will be specifically stated when this single patient was excluded from the analyses presented in the next section.

The visual field defect was mild in 10 (40\%) and severe in 11 (44\%). In all cases the side of the visual field defect matched the side of the surgery. The BCVA was $1.2 \pm 0.3$ for the right eye and $1.1 \pm 0.3$ for the left eye. 
RTD related optic disc changes In patients with GEE demonstrated a significant decrease of the averaged PRNFL thickness over time in patients with a visual field defect compared to patients without a visual field defect (GEE, $\beta$ $-0.079, p<0.0001$ ). The raw data are summarised in Fig. 3. The figure also demonstrates that the magnitude of pRNFL atrophy was most pronounced in patients who developed a severe visual field defect compared to those who did not $(\beta-0.005, \mathrm{p}=0.0002)$.

The degree of pRNFL atrophy in the first three months was strongly correlated to the extend of tissue resection $(R=-0.69, p=0.0007)$. This relationship was lost after 12 months $(p=0.2)$.

There was no correlative statistical relationship between the degree of PRNFL atrophy and the distance from the retina to the site of surgery at any time-point. Likewise the substance class of the AEDs had no influence on the dynamics of RTD or PRNFL thickness.

RTD related macular changes The relationship between surgery, visual field defect and individual retinal layers in the macular (Fig. 2] was investigated. The individual longitudinal raw data for all patients and the averaged group changes are summarised in Fig. 3. There was a significant degree of macular GCIPL loss for the averaged ETDRS grid $(\beta-0.048, \mathrm{p}=0.01$, Fig. 3 A). This relationship was also found for the $G C L-R O I$ ( $\beta-0.075, p=0.001$, Fig. 4 and the GCIPL-ROI ( $\beta-0.016, \mathrm{p}=0.049)$. For the total macular volume no such changes were observed over time (Fig. 3 D, $\beta-0.009, p=0.261$ ).

The larger the volume of brain tissue resected, the larger was the degree of atrophy. After three months, the correlations were significant for the GCIPLROI ( $R=-0.65, p=0.0007)$ and the $G C L-R O I(R=-0.78, p<0.0001)$, but not the averaged ETDRS GCIPL $(R=0.23, p=0.28)$. The linear relationship between brain volume resected and the degree of atrophy was lost after one year. 
The thickness of the INL remained stable over time (GEE, $\beta-0.052, \mathrm{p}=0.15$, Fig. 3 C). Presence of microcystic macular oedema (MMO) was observed only in one of our patients (Fig. 5). This was the only patient with damage to the optic tract. Therefore this patient was excluded from the following analyses.

The substance class of the AEDs had no influence on the dynamics of RTD in the macula.

Adjusted RTD related retinal layer changes and time course The statistical re-analyses of the 24 patients with true RTD confirmed a significant group difference for the pRNFL $(\beta-0.045, \mathrm{p}=0.0009)$ and $\mathrm{GCL}-\mathrm{ROI}(\beta-0.076$, $\mathrm{p}=0.002)$. There was no group difference for the ETDRS-GCIPL $(\beta-0.035$, $\mathrm{p}=0.11)$ or $\mathrm{GCIPL}-\mathrm{ROI}(\beta-0.011, \mathrm{p}=0.21)$. There has been no change in the significance levels of the correlative relationships between brain tissue resection and retinal layer atrophy.

For all comparisons the magnitude of atrophy was most extensive in patients with a severe visual field defect. The atrophy rate slowed down after three months in patients with a small visual field defect (Fig. 6] A), but continued for up to a year in patients with a more severe visual field defect (Fig. 6 B).

The degree of atrophy correlated with the extend of brain tissue resection (Fig. 6 C). The correlation was inverse for the GCIPL-ROI $(R=-0.44, p=0.03)$ and the $G C L-R O I(R=-0.67, p=0.0004)$, but not the averaged ETDRS GCIPL $(R=0.23, p=0.28)$ at three months. This linear relationship disappeared at month 12 .

Regarding the time course of RTD in the first three months window there was a significant correlation of the atrophy rate with time from surgery for the GCL-ROI ( $R=-0.74, p<0.0001)$ and the GCIPL-ROI $(R=-0.51, p<0.0001$, Fig. 6C). The correlation was statistically weak for the ETDRS-GCIPL (R=$0.23, p=0.03)$ and not significance for the $P R N L(R=-0.11, p=0.23)$. 


\section{Discussion}

This study closes the gap of conclusive evidence to demonstrate that acquired RTD in humans truly exists. The design of this prospective longitudinal study permitted to circumvent crucial confounders which have affected previous work (Goto et al. 2016 Jindahra et al. 2009. Mehta and Plant 2005. Park et al. 2013 Schwartz et al. 2017). In all patients a baseline scan was acquired prior to the intervention. There was precise data on timing of the intervention. There was accurate data on location and volume of brain tissue resection. There was no contamination of the process of acquired RTD by other pathologies. This permitted us to report two novel observations.

First, by combination of above data we were able to demonstrate that the extend and time course of acquired RTD is related to the volume and location of brain tissue resection. A larger volume of brain tissue loss causes more extensive damage to Meyer's loop compared to smaller areas of resection. Consequently, with larger brain tissue loss acquired RTD results in atrophy of a larger retinal area compared to what is observed with smaller tissue resections. The relationship between brain tissue volume loss and inner retinal layer atrophy is linear and highly significant. The area of atrophy is determined by the size of damage to the retina-cortical projections.

Second, there is no significant difference in the initial speed of acquired RTD in patients with large compared to those with small areas of brain tissue resection. There is however a difference in the longterm duration of acquired RTD. With smaller areas of brain tissue resection, acquired RTD slowed down within about three months. In contrast acquired RTD continued for at least one year in patients with larger areas of brain tissue resection, but remained strictly within the area of the retina-cortical projections. This observation is difficult to explain with a simple mechanistic model of axonotmesis. We therefore interprete our data on the biological background of the known prolonged time 
course of axonal degeneration in the human brain is prolonged when compared to the peripheral nervous system (Stoll et al. 1989a: Stoll et al. 1989b). In the brain axonal degeneration can continue for several years. One additional mechanistic factor which will need to be considered relates to divergence on convergence of the retino-cortico axonal projections. We speculate about the existence of a potentially salvageable penumbra area to RTD. One method to investigate this further are MRI tractography and DTI sequences including fractional anisotropy.

In this context other spatial cellular and molecular factors require discussion. The "special geometry and spatial relation of axons and oligodendrocytes" has been applied to "help to explain the spreading of pathology" in a different disease, multiple sclerosis (Simons et al. 2014). Then there are a number of molecular mechanisms which influence axonal degeneration (Conforti et al. 2014 Freeman 2014). Next, in order to permit for saltatory conduction, axons have become dependent on oligodendrocytes. Consequently, axons are electrically and metabolically isolated (Nave 2010). This dependence has been eloquently presented as the "virtual hypoxia" hypothesis (Trapp and Stys 2009). Simons et al suggest that the greater variability of degeneration in the CNS depends on the stoichiometry of the axon-oligodendrocyte relationship which varies in different parts of the brain. There is prospective data to suggest that such a relationship can be tested in vivo by combining body fluid biomarkers with MRI and retinal OCT (Petzold et al. 2015). Particularly neurofilament proteins, specific biomarkers for neurodegeneration, have advanced the field (Khalil et al. 2018: Petzold 2005). It has been proposed that with RTD "understanding this time-lag may potentially open a new therapeutic window for future neuroprotective strategies." (Petzold 2014). Present data can further strengthen this line of argumentation if existence of a salvageable penumbra to RTD can be shown. 
Extending on above line of argumentation, we can confirm the observation on more rapid development of inner retinal layer atrophy due to direct retrograde axonal degeneration, illustrated by one of our patients (Fig. 5), rather than truly acquired RTD (Jindahra et al. 2012). The faster degeneration follows direct axonal injury for example by damage to the LGN. Jindahra et al. correctly point out that this may explain the more rapid and severe development of pRNFL atrophy in their patient \#11 (Jindahra et al. 2012). Anatomically the optic tract is only separated by a very narrow choroid fissure from the hippocampus (Kiernan 2012). Damage to the optic tract at this location had occurred in one of our patients (Fig. 5). Consistent with the observation by Jindahra et al. inner retinal layer atrophy was quickest and most severe in this patient. Direct rather then trans-synaptic retrograde axonal degeneration occurred. Likewise the presence of INL swelling and MMO in this case was consistent with the histological observations by van Buren (Buren 1963). Only experimental trans-section of the anterior visual pathways caused the combination of RNFL and GCIPL atrophy with INL thickening and presence of microcysts. This pattern of MMO has also been observed with compression of the anterior optic pathways by a tumor (Abegg et al. 2012. Pott et al. 2016). The publications by Jindahra et al on time domain OCT data pre-dated the discovery of MMO which required spectral domain OCT technology (Gelfand et al. 2012). Therefore we do not know whether their case \#11 also had MMO.

To the best of our knowledge MMO has yet to be reported in cases with isolated lesions to the posterior visual pathways. Notably MMO was absent in a case with severe GCIPL atrophy following hemispherectomy for intractable childhood epilepsy (Handley et al. 2017).

The work by Gabilondo and colleagues considered RTD in a in a different disease, multiple sclerosis. These authors also found a quantitative relationship between changes in brain volume and the PRNFL over a 12 months obser- 
vation period (Gabilondo et al. 2013). A $1 \mathrm{~cm}^{3}$ reduction of the visual cortex related to a $0.6 \mu \mathrm{m}$ reduction of the PRNFL (averaged between both eyes). Interestingly these authors found a very mild increase of the macular volume over time, consistent with our observation. It is not known whether or not this was related to thickening of the INL or development of MMO in MS. There is however one more point to consider from the literature on multiple sclerosis. The time course of RTD following optic neuritis. It has been shown that pRNFL atrophy comes to a halt after about 12 months (Costello et al. 2006). This observation is consistent with our study.

In addition to these fundamental observations our study also makes a new methodological point. The data demonstrate the superiority of using region specific (ROI) analysis of the retina over the standard ETDRS grid. This is to the best of our knowledge the first study to employ ROI analysis targeted at the retinotopic projections from the retina to the visual cortex. There were strong correlations between brain tissue loss and the degree of GCL-ROI and GCIPL-ROI atrophy. More brain tissue loss was related to a greater degree of retinal atrophy. In contrast, no such relationships were found for the ETDRS grid sectors. The ETDRS had been developed as a standard for treatment trials in diabetic retinopathies. The ETDRS grid does not provide a good metric for neurological disease because the sectors cross the relevant vertical and horizontal meridian of the retina (Fig. 2). Taken together future studies can be improved by use of ROI specific analysis of OCT atrophy data.

This observation is consistent with two recent methodological papers on ROI analyses in glaucoma research (Wu et al.2018ab). Comparable to present data these authors also used manual and automated ROI analysis. Importantly, the retest variability for ROI analysis was excellent at $\pm 1.3 \mu \mathrm{m}$. (Wu et al. 2018b). As in study manual ROI analysis was superior to automated $\mathrm{ROI}$ analysis for longitudinal detection of macular inner retinal layer atrophy. 
Finally, comparison of Figures 3 and 4 shows much smaller confidence limits for the mean predicted values (grey shaded areas in the figures) for the ROI concept compared to the conventional EDTRS grid.

There are also limitations to our study which require consideration. The estimation of the time course of RTD was limited by the frequency of measurements. This was due to striking a balance between the need for longitudinal observations and ethical concerns to call patients back to frequently after an invasive brain procedure. Retrospectively, the rapid development of atrophy already within three months does indicate that future studies will need to plan for more frequent OCT assessments in the first three months. Higher frequency follow-up assessment may however increase patient drop-out which had been a problem with the last follow-up appointment in this study.

Another potential criticism is that we did not analyse retinal layers below the INL. There are three main reasons for this. First RTD from the brain to the eye stops at the INL (Petzold 2016. Petzold et al. 2017). Second, quantitative assessment of the outer retinal layer thicknesses has not yet reached the same level of accuracy to what can be done with inner retinal layers (Oberwahrenbrock et al. 2015). Third, axonal convergence and divergence modulate, at least in the context of anterograde trans-synaptic degeneration, propagation through the INL (Panneman et al. 2019). This process is suspected to depend on the geography area of atrophy. Investigation of such relationships will require new ROI approaches and algorithms, capable to precisely delineate geographic areas. These algorithms will need to be developed for future studies.

Taken together our data suggest that there are three patterns of inner retinal layer atrophy observed. First, the fastest and most devastating is caused by direct retrograde axonal degeneration which was in this study associated with MMO. Second, acquired RTD due to small lesions is slightly slower and 
remains restricted to the approximate area of damage to the retino-cortical projections. Third, acquired RTD due to large lesions continues for a prolonged time, radiates out of the area of immediate damage and is governed by the special spacial stoichiometric relationship of the axon-oligodendrocyte interface.

A better understanding of these relationships will be important because acquired RTD may be a more suitable target for neuroprotection than Wallerian degeneration (Petzold 2014). It would be interesting to study if neuroprotective ion channel blockers such as Phenytoin (Raftopoulos et al. 2016), Lamotrigine (Kapoor et al. 2010) or novel treatment approaches in epilepsy (Kätzel et al.2014 Lieb et al.2018) can improve visual outcome after epilepsy surgery. 


\section{Contributors}

WAEJV: conceived the idea for this study, preformed clinical assessments, evaluated the ophthalmological data, contributed to the literature research, contributed to the statistical analyses and revised the manuscript. JCB: conceived the idea for this study, recruited and consented patients, evaluated the neuro-surgical data, revised the manuscript. AP: conceived the idea for this study, performed the literature search, evaluated OCT data, performed the statistical analysis and wrote the first draft of the manuscript.

\section{Declaration of interest}

\section{Acknowledgement}

We are grateful to Heidelberg Spectralis for the purpose build $8 \times 8$ square grid which at least in part resulted from discussions related to this study. We are grateful for logistic support to Mrs S van Went and to Mrs L Verlaat.

\section{Funding}

This study was not funded. AP was supported by the National Institute for Health Research (NIHR) Biomedical Research Centre based at Moorfields Eye Hospital NHS Foundation Trust and UCL Institute of Ophthalmology. The views expressed are those of the author(s) and not necessarily those of the NHS, the NIHR or the Department of Health.

\section{Data Availability Statement}

The data that support the findings of this study are available from the corresponding author, upon reasonable request and in accordance with GDPR regulations. 


\section{Conflicts of interest}

AP reports that the VUmc MS Centre Amsterdam participated in the OCTIMS trial and the centre has received research support for OCT projects from the Dutch MS Society.

WAdVK and JCB report no conflict of interest.

\section{References}

Abegg, M., M. Zinkernagel, and S. Wolf (2012). "Microcystic macular degeneration from optic neuropathy". Brain 135, e225-e225. DOI: 10.1093/ brain/aws215

Balk, L. J. and A. Petzold (2013). "Influence of the eye-tracking-based followup function in retinal nerve fiber layer thickness using fourier-domain optical coherence tomography." Invest Ophthalmol Vis Sci 54, p. 3045. DOI: 10.1167/iovs.13-12073.

Buren, J. van (1963). "Trans-synaptic retrograde degeneration in the visual system of primates". J Neurol Neurosurg Neuropsych 26, pp. 402-409. DOI: 10.1136/jnnp.26.5.402.

Clayton, L. M. S., J. S. Duncan, S. M. Sisodiya, and J. F. Acheson (2010). "Delayed, rapid visual field loss in a patient after ten years of vigabatrin therapy." Eye (Lond) 24, pp. 185-186. Dol: 10.1038/eye.2009.64.

Clayton, L. M., M. Dévilé, T. Punte, C. Kallis, et al. (2011). "Retinal nerve fiber layer thickness in vigabatrin-exposed patients." Ann Neurol 69, pp. 845854. DOI: 10.1002 /ana.22266.

Conforti, L., J. Gilley, and M. P. Coleman (2014). "Wallerian degeneration: an emerging axon death pathway linking injury and disease". Nature Reviews Neuroscience 15, p. 394. DOI: 10.1038/nrn3680.

Costello, F., S. Coupland, W. Hodge, G. R. Lorello, et al. (2006). "Quantifying axonal loss after optic neuritis with optical coherence tomography." Ann Neurol 59, pp. 963-969. Dol: 10.1002/ana.20851.

Cruz-Herranz, A., L. J. Balk, T. Oberwahrenbrock, S. Saidha, et al. (2016). "The APOSTEL recommendations for reporting quantitative optical coherence tomography studies." Neurology 86, pp. 2303-2309. DOI:10.1212/ WNL.0000000000002774.

Freeman, M. R. (2014). "Signaling mechanisms regulating Wallerian degeneration". Current opinion in neurobiology 27, pp. 224-231. DOI: 10.1016/ j.conb.2014.05.001.

Gabilondo, I., M. Sepulveda, S. Ortiz-Perez, E. Fraga-Pumar, et al. (2013). "Retrograde retinal damage after acute optic tract lesion in MS". J Neurol Neurosurg Psychiatry 84, pp. 824-826. DOI: 10.1136/jnnp-2012304854 . 
Gelfand, J. M., R. Nolan, D. M. Schwartz, J. Graves, and A. J. Green (2012). "Microcystic macular oedema in multiple sclerosis is associated with disease severity." Brain 135, pp. 1786-1793. Dol: 10.1093/brain/aws098.

Goto, K., A. Miki, T. Yamashita, S. Araki, et al. (2016). "Sectoral analysis of the retinal nerve fiber layer thinning and its association with visual field loss in homonymous hemianopia caused by post-geniculate lesions using spectraldomain optical coherence tomography". Graefe's Archive for Clinical and Experimental Ophthalmology 254, pp. 745-756. DOI: 10.1007/s00417015-3181-1.

Handley, S. E., V. S. Panteli, and A. Liasis (2017). "Trans-synaptic Retrograde Degeneration Following Hemispherectomy in Childhood." Neuroophthalmology (Aeolus Press) 41 (2), pp. 103-107. ISSN: 0165-8107. DOI: $10.1080 / 01658107.2016 .1276935$,

Jeelani, N. U. O., P. Jindahra, M. S. Tamber, T. L. Poon, et al. (2010). "Hemispherical asymmetry in the Meyerś Loop: a prospective study of visualfield deficits in 105 cases undergoing anterior temporal lobe resection for epilepsy." J Neurol Neurosurg Psychiatry 81, pp. 985-991. DOI: 10.1136/jnnp.2009.182378.

Jindahra, P., A. Petrie, and G. T. Plant (2009). "Retrograde trans-synaptic retinal ganglion cell loss identified by optical coherence tomography." Brain 132, pp. 628-634.

Jindahra, P., A. Petrie, and G. T. Plant (2012). "The time course of retrograde trans-synaptic degeneration following occipital lobe damage in humans." Brain 135, pp. 534-541. Dol: 10.1093/brain/awr324.

Kapoor, R., J. Furby, T. Hayton, K. J. Smith, et al. (2010). "Lamotrigine for neuroprotection in secondary progressive multiple sclerosis: a randomised, double-blind, placebo-controlled, parallel-group trial." The Lancet Neurology 9 (7), pp. 681-688. ISSN: 1474-4465. DOI: $10.1016 / \mathrm{s} 1474-$ 4422(10)70131-9.

Kätzel, D., E. Nicholson, S. Schorge, M. C. Walker, and D. M. Kullmann (2014). "Chemical-genetic attenuation of focal neocortical seizures". Nature Communications 5, p. 3847. ISSN: 2041-1723. DOI: 10.1038/ncomms4847.

Khalil, M., C. E. Teunissen, M. Otto, F. Piehl, et al. (2018). "Neurofilaments as biomarkers in neurological disorders." Nature Reviews Neurology 14, pp. 577-589. ISSN: 1759-4766. DOI: 10.1038/s41582-018-0058-z.

Kiernan, J. (2012). "Anatomy of the temporal lobe". Epilepsy research and treatment 2012, p. 176157. DOI: 10.1155/2012/176157.

Lieb, A., Y. Qiu, C. L. Dixon, J. P. Heller, et al. (2018). "Biochemical autoregulatory gene therapy for focal epilepsy." Nature medicine 24 (9), pp. 13241329. ISSN: 1546-170X. DOI: 10.1038/s41591-018-0103-x.

Luco, C., A. Hoppe, M. Schweitzer, X. Vicuna, and A. Fantin (1992). "Visual field defects in vascular lesions of the lateral geniculate body." Journal of Neurology, Neurosurgery \& Psychiatry 55, pp. 12-15. DOI: 10.1136/ jnnp.55.1.12. 
Mehta, J. S. and G. T. Plant (2005). "Optical Coherence Tomography (OCT) Findings in Congenital/Long-Standing Homonymous Hemianopia". Am J Ophthalmol 140, pp. 727-729. DOI: $10.1016 / \mathrm{j}$.ajo.2005.03.059.

Nave, K.-A. (2010). "Myelination and the trophic support of long axons". Nature Reviews Neuroscience 11, pp. 275-283. DOI: 10.1038/nrn2797.

Oberwahrenbrock, T., M. Weinhold, J. Mikolajczak, H. Zimmermann, et al. (2015). "Reliability of Intra-Retinal Layer Thickness Estimates." PLoS One 10, e0137316. Dol: 10.1371/journal.pone.0137316.

Panneman, E., D. Coric, L. Tran, W. de Vries-Knoppert, and A. Petzold (2019). "Progression of Anterograde Trans-Synaptic Degeneration in the Human Retina Is Modulated by Axonal Convergence and Divergence". Neuro-Ophthalmology. [in press], pp. 1-9. DOI: 10.1080/01658107.2019.1599027.

Park, H.-Y. L., Y. G. Park, A.-H. Cho, and C. K. Park (2013). "Transneuronal Retrograde Degeneration of the Retinal Ganglion Cells in Patients with Cerebral Infarction". Ophthalmology 120, pp. 1292-1299. DOI: 10.1016/j.ophtha.2012.11.021.

Petzold, A. (2005). "Neurofilament phosphoforms: Surrogate markers for axonal injury, degeneration and loss". J Neurol Sci 233, pp. 183-198. DOI: $10.1016 / j \cdot j n s .2005 .03 .015$.

- (2014). "Neurodegeneration and Multiple Sclerosis". Neurodegenerative Diseases. Springer London, pp. 227-245. DOI: 10.1007/978-1-44716380-0_14.

- (2016). "An early case of a natural barrier to axonal degeneration." Journal of neurology 263 (11), pp. 2330-2331. ISSN: 1432-1459. DOI: 10 . 1007/s00415-016-8289-7.

Petzold, A., L. J. Balcer, P. A. Calabresi, F. Costello, et al. (2017). "Retinal layer segmentation in multiple sclerosis: a systematic review and metaanalysis." The Lancet. Neurology 16 (10), pp. 797-812. ISSN: 14744465. DOI: 10.1016/S1474-4422(17)30278-8.

Petzold, A., P. G. Nijland, L. J. Balk, A. M. Amorini, et al. (2015). "Visual pathway neurodegeneration winged by mitochondrial dysfunction." Ann Clin Transl Neurol 2, pp. 140-150. DOI: 10.1002/acn3.157.

Petzold, A., M. P. Wattjes, F. Costello, J. Flores-Rivera, et al. (2014). "The investigation of acute optic neuritis: a review and proposed protocol." Nat Rev Neurol 10, pp. 447-458. DOI:10.1038/nrneurol.2014.108.

Pott, J. W. R., W. A. E. J. de Vries-Knoppert, and A. Petzold (2016). "The prevalence of microcystic macular changes on optical coherence tomography of the macular region in optic nerve atrophy of non-neuritis origin: a prospective study." The British journal of ophthalmology 100 (2), pp. 216221. ISSN: 1468-2079. DOI: 10.1136/bjophthalmol-2014-305737.

Pula, J. H. and C. A. Yuen (2017). "Eyes and stroke: the visual aspects of cerebrovascular disease". Stroke and Vascular Neurology 2, pp. 210-220. DOI: 10.1136/svn-2017-000079.

Raftopoulos, R., S. J. Hickman, A. Toosy, B. Sharrack, et al. (2016). "Phenytoin for neuroprotection in patients with acute optic neuritis: a randomised, 
placebo-controlled, phase 2 trial." Lancet Neurol 15, pp. 259-269. DOI: 10.1016/S1474-4422(16)00004-1.

Schippling, S., L. Balk, F. Costello, P. Albrecht, et al. (2015). "Quality control for retinal OCT in multiple sclerosis: validation of the OSCAR-IB criteria." Mult Scler 21, pp. 163-170. DOI: 10.1177/1352458514538110.

Schwartz, S. G., A. Monroig, and H. W. Flynn (2017). "Progression of Transsynaptic Retinal Degeneration With Spectral-Domain Optical Coherence Tomography." American journal of ophthalmology case reports 5, pp. 67-72. ISSN: 2451-9936. DOI: 10.1016/j.ajoc.2016.12.010.

Simons, M., T. Misgeld, and M. Kerschensteiner (2014). "A unified cell biological perspective on axon-myelin injury." J Cell Biol 206, pp. 335-345. DOI: $10.1083 / \mathrm{jcb} .201404154$.

Stoll, G., J. W. Griffin, C. Y. Li, and B. D. Trapp (1989a). "Wallerian degeneration in the peripheral nervous system: participation of both Schwann cells and macrophages in myelin degradation". J Neurocytol 18, pp. 671-683. DOI: $10.1007 / \mathrm{bf01187086.}$

Stoll, G., B. Trapp, and J. Griffin (1989b). "Macrophage function during Wallerian degeneration of rat optic nerve: clearance of degenerating myelin and la expression". J Neurosci 9, pp. 2327-2335. DOI: 10.1523/jneurosci.0907-02327.1989.

Tewarie, P., L. Balk, F. Costello, A. Green, et al. (2012). "The OSCAR-IB Consensus Criteria for Retinal OCT Quality Assessment." PLoS One 7, e34823. Dol: $10.1371 /$ journal.pone.0034823.

Trapp, B. D. and P. K. Stys (2009). "Virtual hypoxia and chronic necrosis of demyelinated axons in multiple sclerosis." Lancet Neurol 8, pp. 280-291. DOI: 10.1016/S1474-4422(09)70043-2.

Wu, Z., A. Thenappan, D. S. D. Weng, R. Ritch, and D. C. Hood (2018a). "Detecting Glaucomatous Progression With a Region-of-Interest Approach on Optical Coherence Tomography: A Signal-to-Noise Evaluation". Translational Vision Science \& Technology 7, p. 19. Dol: 10.1167/tvst.7.1.19.

Wu, Z., D. S. D. Weng, A. Thenappan, R. Ritch, and D. C. Hood (2018b). "Evaluation of a Region-of-Interest Approach for Detecting Progressive Glaucomatous Macular Damage on Optical Coherence Tomography". Translational Vision Science \& Technology 7, p. 14. DOI: 10.1167/tvst.7.2.14. 
Figure 1: Longitudinal Goldmann visual fields taken at $(A, B)$ baseline, $(C, D)$ three months and $(E, F)$ one year after epilepsy surgery. The cSLO image of the fundus at baseline was overlaid such that the foveola located to the central field and the optic disc located over the blind spot. To the right of each image the data for the $p R N F L$ are shown as inset. The inset shows the location of the peripapillary ring scan (green) around the optic disc in the CSLO image. The corresponding OCT B-scan is shown together with the automated segmentation lines (red lines) for the pRNFL and the corresponding thickness values in form of a bar chart (left of inset) and thickness plot (right of inset). Next the post-surgery MRI brain scan shows extend of temporal lobectomy with measurements of anterior visual path lengths to site of lesion. The time series to the left $(A, C, E)$ shows a patient who developed a small left superior quadrantanopia following partial right temporal lobectomy. In this patient OCT did not permit to detect any pRNFL or macular GCIPL atrophy. The time series to the right $(B, D, E)$ shows a severe right superior quadrantanopia following a left partial temporal lobectomy. The OCT shows clearly the development of pRNFL atrophy and hemiatrophy of the macular GCIPL (green area in the OCT overlay thickness map), exactly matching the central visual field defect.

Figure shown on next page. 

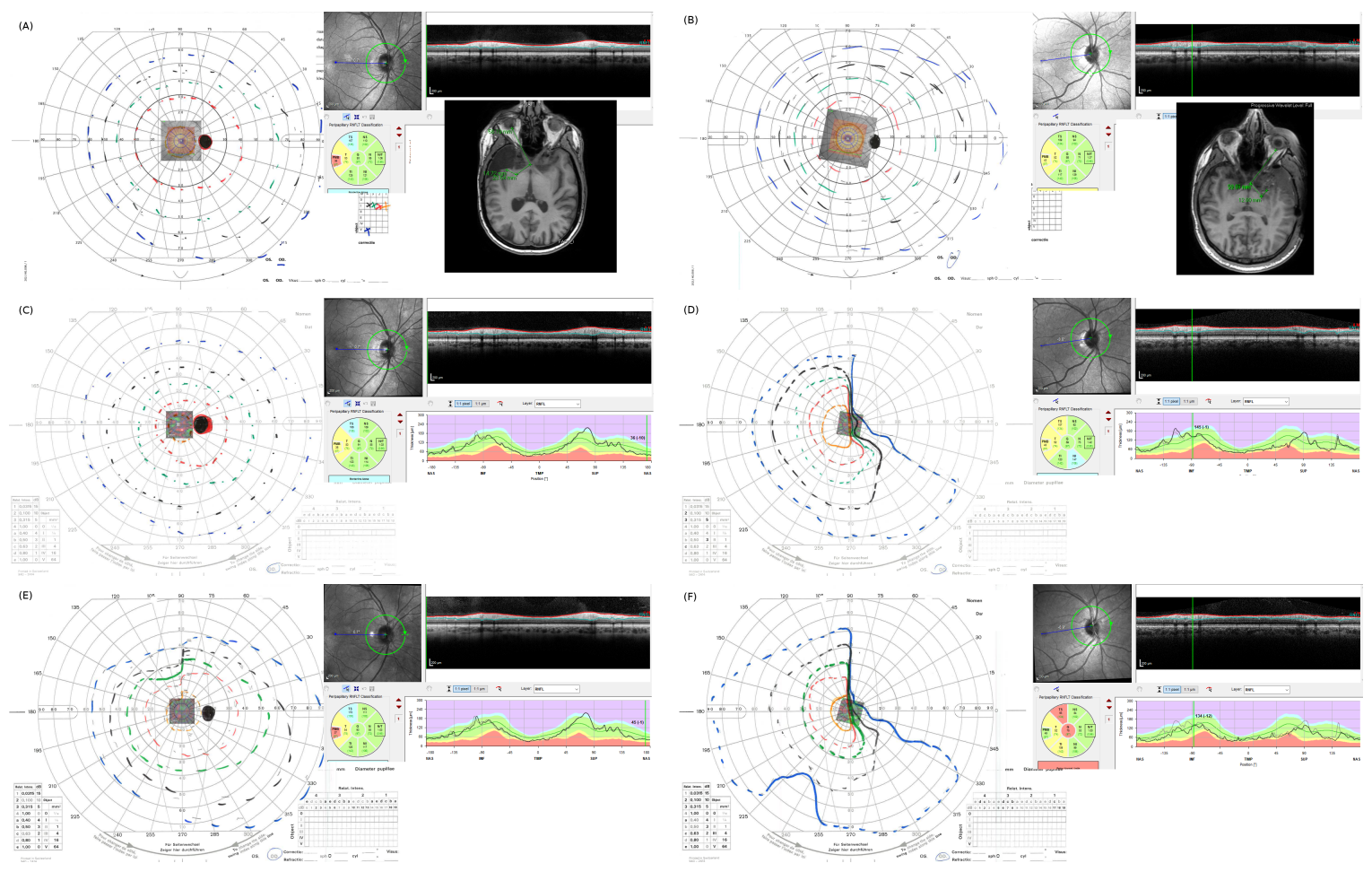

Page 24 
Figure 2: Explanation of the rationale for the ROI concept used in this study. Until now the ETDRS grid has almost exclusively been used in the OCT literature as a matrix for defining areas of atrophy in the macula. The thickness of retinal layers is shown by a heat map where the brightness of the colour overlay indicates the thickness of a specific retinal layer. The heat map is shown as an overlay to the scanning laser ophthalmoscopic image (grey) of the fundus. In this figure yellow colouring in the heat map indicates a thicker GCIPL compared to the darker orange colouring. The individual OCT B-scans are now shown in this figure, but typical examples are presented in figures 3 and 5. (A) By convention the sectors of the EDTRS are labelled as follows: central $(=C$, yellow letter), inner nasal $(=N 1$, black letters), outer nasal $(=N 2)$, inner superior (S1), outer superior (S2), inner temporal (T1), outer temporal (T2), inner inferior (I1) and outer inferior (I2) sector. The reporting of these EDTRS sectors is the basis to all previous studies on RTD we had reviewed. (B) An important limitation of the ETDRS grid is however that conventional sectoral averaging over an area which crosses the vertical midline $(S 1, S 2,11,12)$ masks relevant data on localised atrophy (yellow arrows). An example is shown for an area of interest (ROI, green arrow) where atrophy of the GCL (darker colour in the heat map) can be observed following injury to Meyer's loop during surgery which also cause a superior quadrantanopia in the patients field of vision. (C) For this reason we used a pointer (green arrows) and a purpose build $8 \times 8$ box grid (green ellipsoid area) to get individual retinal layer thickness data from the ROI. (D) Illustrates how using the ROI concept permits to much more accurately capture localised atrophy compared to the EDTRS grid. In this example the development of temporal atrophy would have been completely missed by using data from the temporal sectors of the EDTRS grid (T1, T2). The white numbers inside the squares show the thickness and volume data for the GCL. For the innermost box of the green highlighted ROI, the GCL thickness averaged at $41 \mu \mathrm{m}$ at baseline and was only $31 \mu \mathrm{m}$ at follow-up. Similar data are available for all other segmented retinal layers.

Figure shown on next page. 


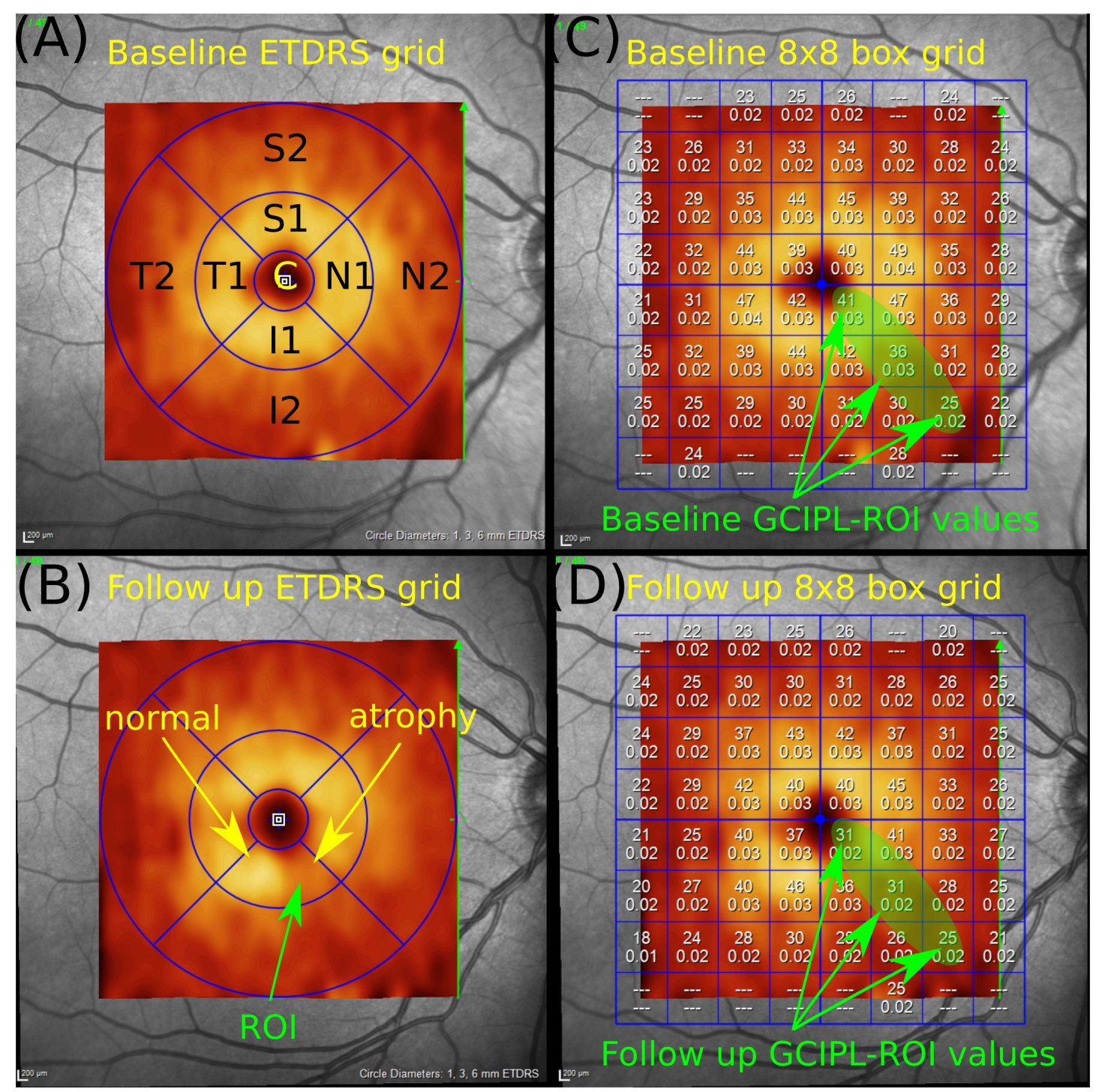

Page 26 
Figure 3: The longitudinal profile of (A) the pRNFL, (B) GCIPL, (C) INL, (D) macular volume (MV). The OCT data come from the conventional ETDRS grid (see Figure 2 A). In each graph the top panel shows data from patients with normal visual fields, the middle panel from patients with any mild type of visual field defect following surgery and the bottom panel from those patients who developed a severe visual field defect after surgery for epilepsy. All data are presented separately for the right (OD) and left (OS) eyes. For each retinal layer the regression over time is indicated by a bold black line together with the confidence limits for the mean predicted values (grey shaded area). Individual patient data are tracked by light grey lines. The $y$-axis shows individual retinal layer thicknesses as $\mu m$ and the $x$-axis the time from baseline (left vertical reference line), three months and twelve months follow-up (right vertical reference line).

Figure shown on next page. 
Region of interest specific retinal OCT layers changes in relation to the visual field

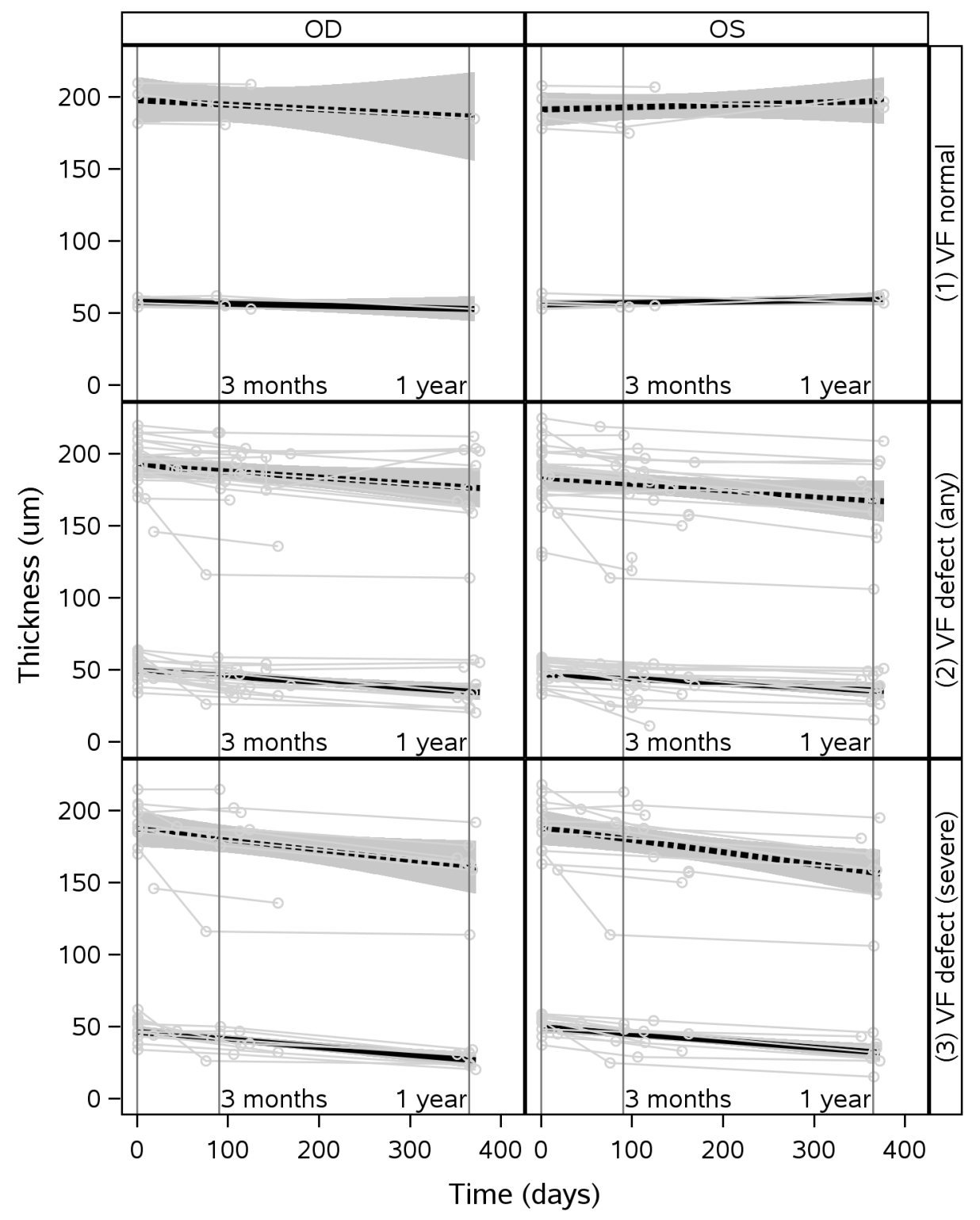


Figure 4: The longitudinal change of the thickness in the macular ROI following surgery for epilepsy. The GCIPL (ROI $8 \times 8$ square grid) and GCL (VF loss matched ROI) thicknesses are shown. The top row shows data for patients with normal visual fields. The bottom row presents data for patients who developed a visual field defect following surgery. Data are presented separately for the right $(R)$ and left (L) eye. For each retinal layer the regression over time is indicated. There is a significant loss of the GCIPL (ROI) over time ( $p<0.0001)$ in the macular area corresponding to site of surgery, but not in patients without visual field defect $(p=0.27)$. Likewise there is a significant loss of the GCL (ROI) in the area matching the visual field defect on Goldmann perimetry when compared to patients without visual field defect $(p<0.0001)$. The shaded areas indicate the 95\% confidence limits for the regression. The $y$-axis shows individual retinal layer thicknesses as $\mu \mathrm{m}$ and the $x$-axis the time from baseline (left vertical reference line), three months and twelve months follow-up (right vertical reference line).

Figure shown on next page. 
(A)

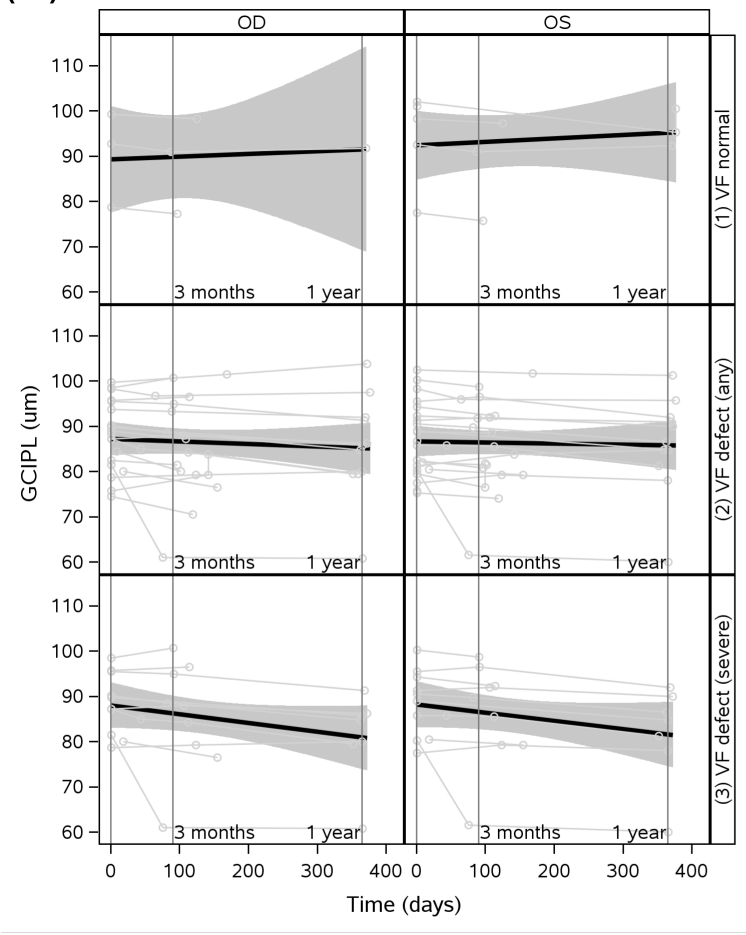

(C)

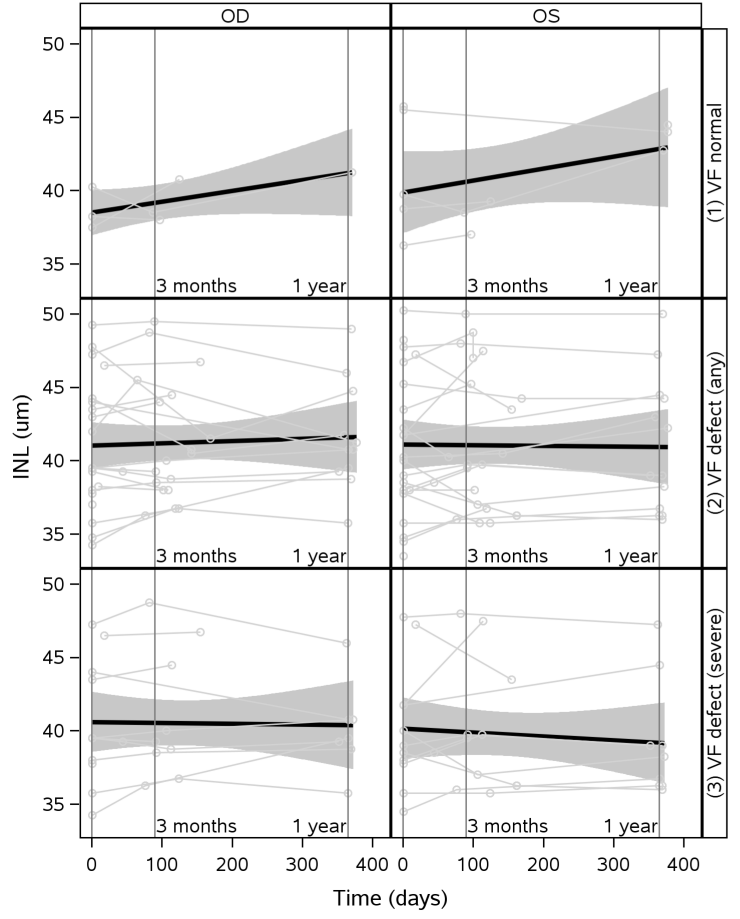

(B) tra

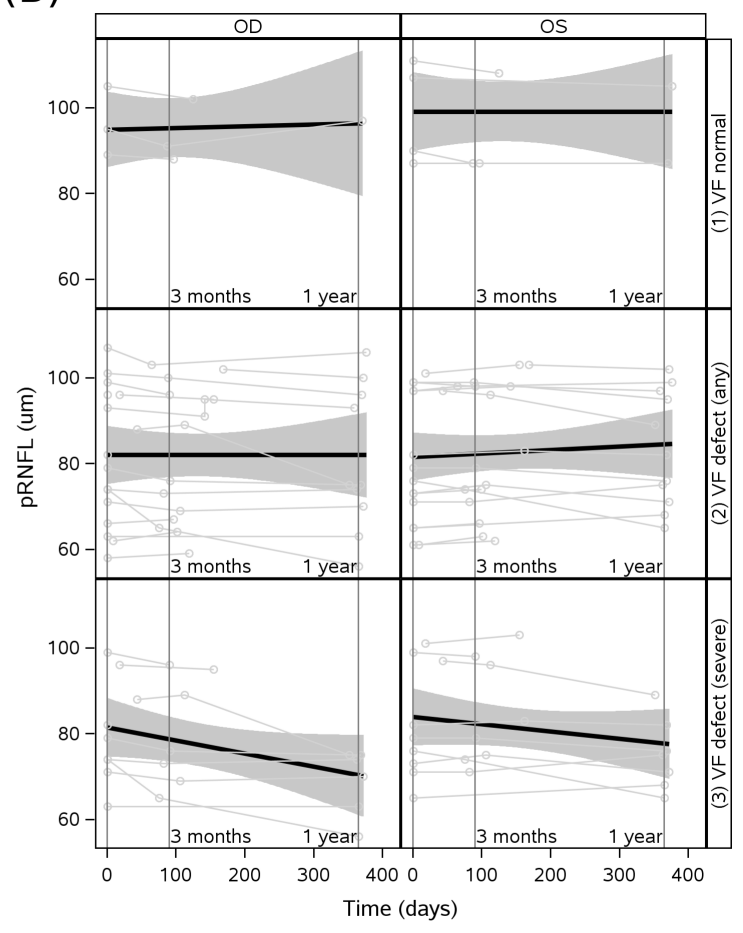

(D)

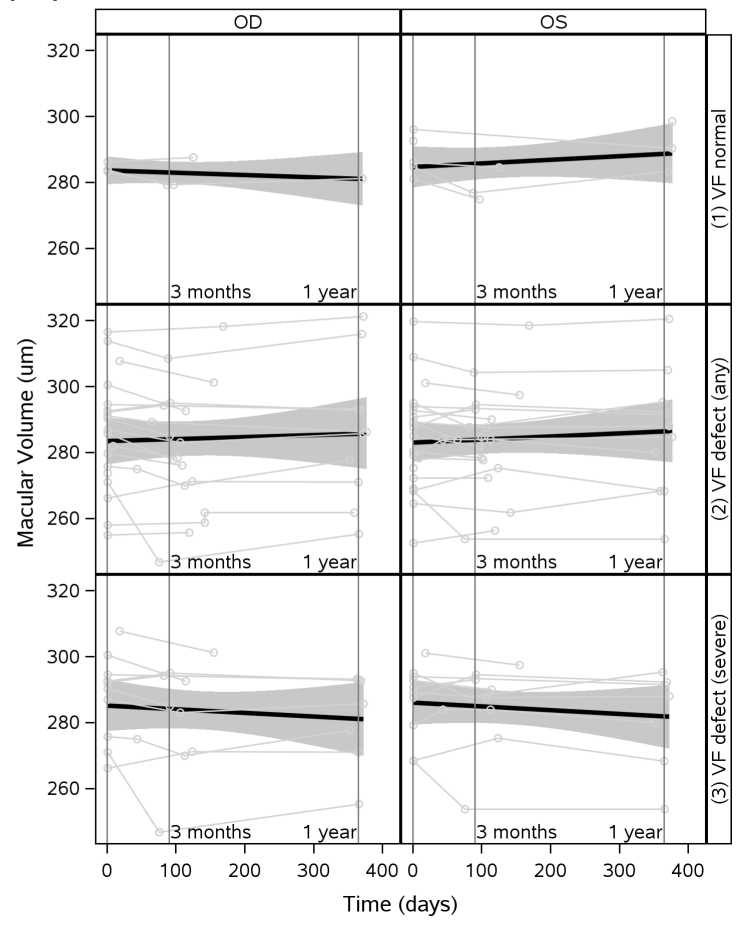

Page 30 
Figure 5: Time course of GCL atrophy, INL thickening and MMO appearance in a 29 year old woman following partial temporal lobe resection. She developed a homonymous right hemianopia (A) OS, (B) OD. (C) The baseline macular OCT scan had a normal thickness of the GCL. (D) After three months there was hemi-macular GCL atrophy ("half moon sign"). (E) GCL atrophy continued to progress mildly one year later. (F) The amount of yearly atrophy from baseline is indicated by a green thickness map. (G) AT baseline the INL was of normal thickness. (H) INL volume increase was observed at month three. (I) The INL volume continued to increase and MMO became visible after one year. (J) The amount of annual INL increase is indicated by a red thickness map. (K) the baseline CSLO image of the macular. (L) After one year there were perifoveal (semi) circular abnormal reflexes on CSLO imaging. (M) The baseline OCT B-scan shows normal retinal layer configuration. (N) After one year there was marked atrophy of the RNFL, GCIPL and adjacent thickening of the INL with presence of MMO (arrow heads). All OCT images shown are from the right eye. The images from the left eye exactly match the observed pattern. Summary thickness data for individual sectors of the ETDRS grid are presented to the left as absolute numbers and colour coded thickness map. Numeric data refers to averaged thickness in micrometers (black letters) and averaged volume data in $\mathrm{mm}^{3}$ (red letters). The green arrow in the cSLO image indicates the retinal location of the adjacent OCT B-scan.

\section{Figure shown on next page.}




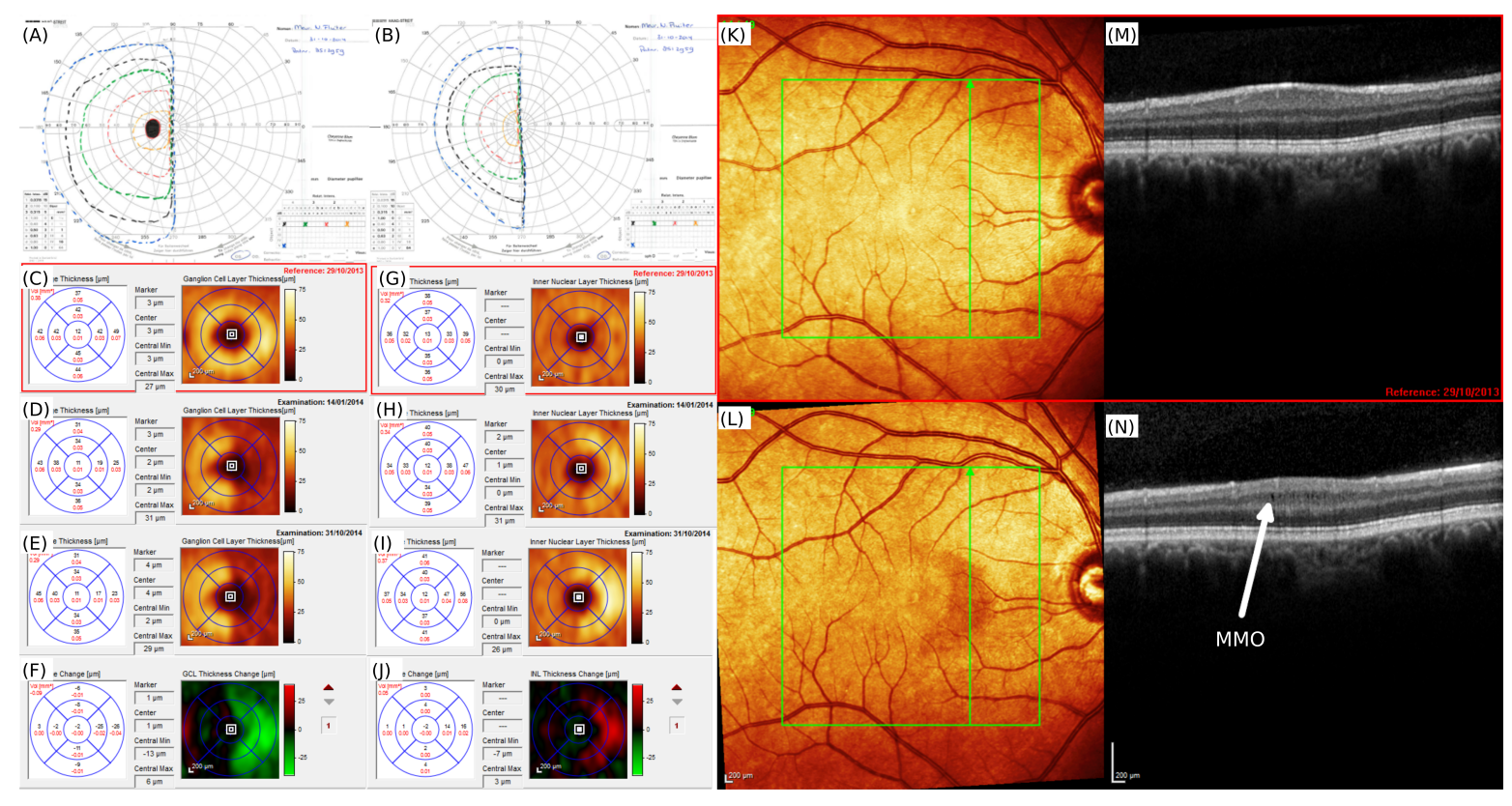


Figure 6: Atrophy data in the 24 patients with true RTD. (A) Atrophy in $\mu \mathrm{m}$ from baseline to month three for the GCL-ROI (large bars) and GCIPL-ROI (small bars, mean $\pm S D$ ). (B) atrophy rate from month three to twelve. (C) Correlation between brain tissue resection and atrophy for the GCL-ROI (solid line, circles) and the GCIPL-ROI (dashed line, crosses) in the three months time window. (D) Time course of atrophy of the GCL-ROI (solid line, circles) and the GCIPL-ROI (dashed line, crosses) for the same period as shown in (A) and $(C)$.

(A)

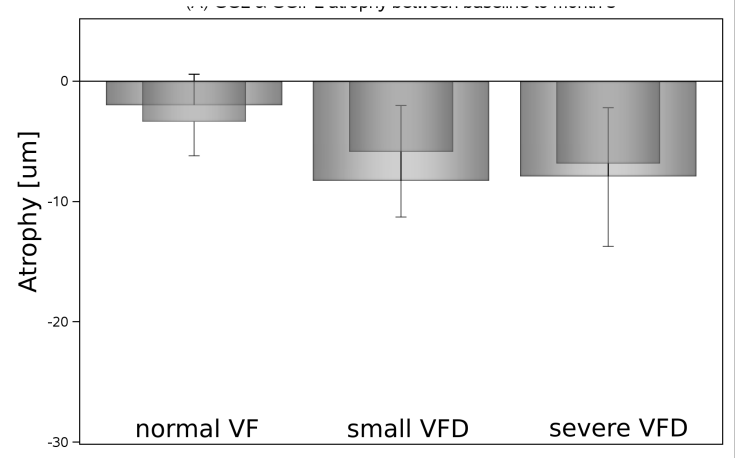

(C)

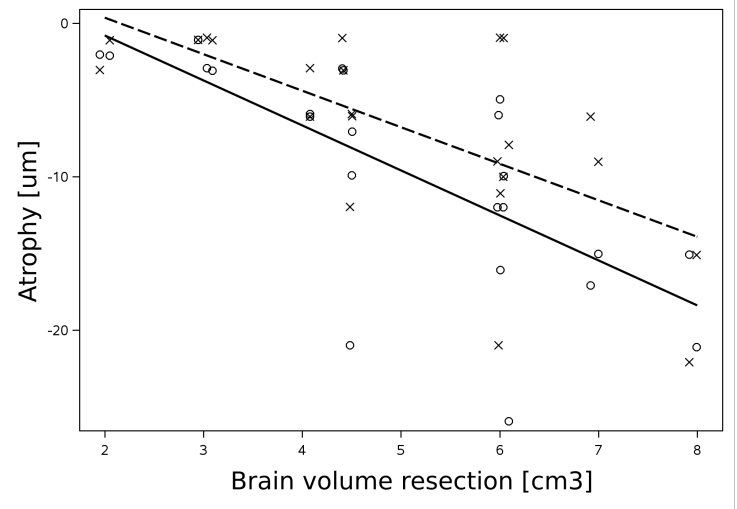

(B)

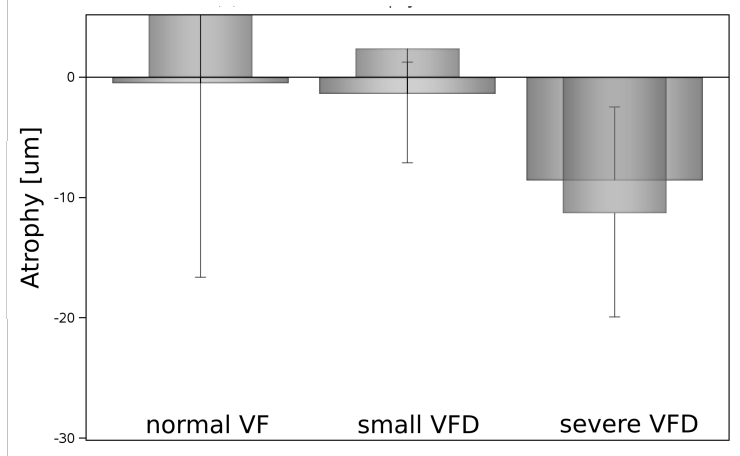

(D)

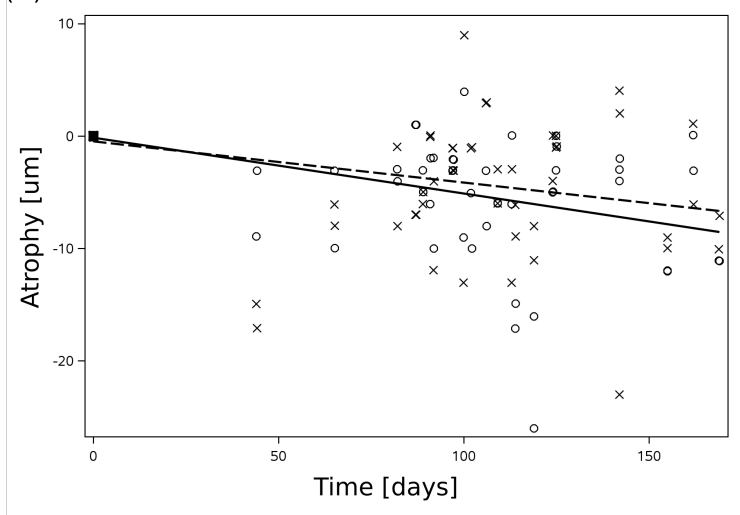

